\title{
ALL: Automated learning laboratory
}

\author{
B. JAMES STARR and SALLY A. FLANIK \\ Howard University, Washington, D.C. 20059
}

\begin{abstract}
ALL is a CAI package designed to give students "hands-on" experience with a primitive learning laboratory. Using ALL, students can serve as either research subjects or experimenters. Both schedules of reinforcement and the nature of the reinforcer may be varied within the program for a number of simple learning tasks.
\end{abstract}

Many of the smaller institutions offering instruction in psychology have neither the budget nor the facilities to support laboratory courses. Nonetheless, many such institutions have access to one or more timesharing terminals. As Green (1972) has pointed out, this milieu is suitable for the use of the computer as an instructional aid. Recent years have witnessed the creation of a variety of computer programs directed toward instruction in various areas of psychology. Lehman, Starr, and Young (1975) reviewed such programs in the teaching of statistics and research methods. Relatively few programs have been developed and disseminated for other areas of psychology (Castellan, Bailey, Lehman, Starr, \& McClelland, Note 1). The present paper briefly describes a few of these endeavors and then describes a program developed by the authors which treats response learning in a computer qua laboratory setting.

Some programs have been developed for use in various experimental psychology courses. An ambitious and apparently successful package of programs for use in a course in cognitive psychology was written by Bewley $(1973,1974)$. Six experiments provide the instructional vehicle for this package. Computer programs, written in timesharing BASIC for use from a Teletype terminal, present the tasks to the students. The students subsequently compete against a model on the task. Students then evaluate their performances vis-à-vis that of the model. The procedures lead the students to experimentation both with features of the model and with other human subjects. The package contains experiments on pattern recognition, shortand long-term memory, concept learning, decision making, and problem solving.

Another important package has been developed by Main (1971). The EXPERSIM program was developed at the University of Michigan to teach students experimental design. The system allows students to engage in repeated experimentation in specific research areas

This work was supported in part by NSF Grant GZ-3431 for a College Faculty Workshop on Computer Science in Social and Behavioral Science Education at the University of Colorado, 1974. B. James Starr is currently on leave at the East-West Center, Honolulu, Hawaii 96822. Thanks are due Maureen Chamberlain for her help in preparing this manuscript. via computer modeling. This permits students to conduct many more "experiments" than they normally would in a typical course. Moreover, the student may actually pursue a "program" of research with regard to a specific area and may learn from the work of others. Thus, the student in many ways enters a microcosm of the world of research. Perhaps the most interesting feature from the viewpoint of the present authors is that the outcomes are modified (i.e., made substantively accurate) with changes in the "real world" state of knowledge in the area. The system currently has imprinting, social facilitation, drug research, etiology of schizophrenia, and motivation as research areas. Others are to be added to this list.

Green (1972) described a variety of programs that provide useful experiences for students in problem solving, concept formation, and decision making. The programs operate on the responses of the students who serve as research subjects. As with the other programs described here, the student not only gains firsthand experience as a research subject (and/or researcher in Bewley's package and EXPERSIM), but he also produces computer output which is useful for didactic purposes.

The authors' own effort, the ALL program, was designed to give beginning students a variety of controlled experiences with principles of response learning in an actual leaming situation. ALL is used to run students through one of several simple learning tasks and record the performance of each individual in two formats. The student receives a record of his performance and the instructor obtains a summary record of the performance of all participants in the experiment.

\section{STIMULI AND TASKS}

ALL's stimuli are (all) numerical. The program prints out two numbers and the student is asked to respond with a third number. Currently, the program comprises four tasks. These elementary tasks are analogs of simple response leaming tasks that have long been used in research on rats and/or college sophomores. The student may receive stimuli where he is required to respond with the number that appears first (or second, i.e., a position learning task). A second task involves the student's responding with an absolute 
item (e.g., the number " 4 " each time). The remaining two tasks pose serial learning problems. The student must respond with the next item in the series. The simpler version of this problem employs an additive or multiplicative (positive or negative) constant to generate the series. The complex version creates the appropriate numerical stimuli by using first an additive constant and then a (different) multiplicative one.

The program can be used to vary both the type of and the schedule of reinforcement. These variables permit manipulation and thus provide a basis for research with regard to their effect on acquisition and extinction of the desired behaviors. Thus, the student may receive positive (RIGHT) or negative (WRONG) reinforcement, or both. In addition, the reinforcement may be continuous or partial, with both fixed- and variable-ratio types available under partial reinforcement. The fixed-ratio schedule may involve the reinforcement of every other (appropriate) trial or every third, fourth, or fifth (appropriate) trial. Reinforcement may also occur on a variable-ratio schedule with a mean fixed by the experimenter.

\section{SUGGESTIONS FOR USE}

Although there were originally two versions of ALL, only one will be detailed here. (Other versions are briefly described below.) The two versions differ only in the way in which the program parameters are selected. In the random version, the task and the type and schedule of reinforcement are all randomly selected. The instructor does not control the selection process. While this version may be advantageous in environments where the program may be run by individuals who lack computer skills, much of the power and instructional value (in terms of controlled experimentation) of this approach would be lost. Therefore, the random version will not be further described here.

The fixed version can easily be used to run experiments. Given the present program structure, a variety of one-factor and higher order investigations are conceivable. The data so generated might provide the springboard for various important experiences. Thus, a number of data analytic skills could be developed or sharpened. Specialized techniques involving curves and curve fitting are applicable. Moreover, the student gains two invaluable experiences. One tangible benefit which accrues is that the student has examined an integrated research venture from start to finish, with relatively little energy expenditure in terms of data collection. In most of his classes the student is exposed only to selected aspects of a research endeavor. Furthermore, the student may also have the advantage of viewing the process from the vantage point of both the experimenter and the research subject.

ALL shares this capacity to offer the student a dualistic experiential research role with Bewley's (1973, 1974) package. It also provides the opportunity for the student to be paced through an orderly progression, going from these roles to a point where he can engage in some programmatic research (as with EXPERSIM) in the area of response learning. ALL is one of few programs focusing on concepts propadeutic to the study of learning. By integrating aspects of computerassisted instruction and process (e.g., experimental) control (Scholz, 1972), it attempts to give the student a flavor of the research process.

\section{DESCRIPTION OF FUNCTION}

The fixed version of ALL permits the instructor or experimenter to specify the experimental conditions prior to a program run. The instructor responds to a number of interrogations by the computer with values for parameters that together determine the nature of the particular investigation. Thus, parameters are entered indicating the type and schedule of reinforcement, the type of task, the number of acquisition trials, the number of extinction trials, the maximum number of subjects to be run under these conditions, and constants to be used in the serial tasks. An additional parameter is used to help generate the starting point for the task, that is, the first stimulus number for the task. An explanation of the input parameters required to initiate runs with ALL is shown in Table 1.

After entry of the parameters, the program spaces the paper, allowing the removal of the information

Table 1

Names of Initializing Input Variables Within the ALL Program and Their Function

\begin{tabular}{|c|c|c|c|c|c|c|}
\hline $\begin{array}{l}\text { Variable } \\
\text { Name } \\
\end{array}$ & \multicolumn{6}{|c|}{ Information Required } \\
\hline $\mathbf{T}$ & \multirow{11}{*}{\multicolumn{6}{|c|}{$\begin{array}{l}\text { Number of acquisition trials desired } \\
\text { Number of extinction trials desired } \\
\text { Number of research subjects to be run } \\
\text { Two-digit number to help generate the first stimulus } \\
\text { Additive constant for certain serial tasks } \\
\text { Constant for use in division on certain serial tasks } \\
\text { Multiplicative constant for certain serial tasks } \\
\text { Number to indicate rate of fixed-ratio partial } \\
\text { reinforcement } \\
\text { Lower limit for rate of variable-ratio reinforcement } \\
\text { Upper limit for rate of variable-ratio reinforcement } \\
\text { Reinforcement option selected (from code list below): }\end{array}$}} \\
\hline $\mathbf{E}$ & & & & & & \\
\hline $\mathbf{N}$ & & & & & & \\
\hline A2 & & & & & & \\
\hline B1 & & & & & & \\
\hline B3 & & & & & & \\
\hline B4 & & & & & & \\
\hline $\mathrm{S}$ & & & & & & \\
\hline \multirow{8}{*}{$\begin{array}{l}\text { S1 } \\
\text { S2 } \\
\text { K1 }\end{array}$} & & & & & & \\
\hline & & & & & & \\
\hline & & & & & & \\
\hline & Reinf & forcement: & Positive & & Negative & Both \\
\hline & \multicolumn{6}{|c|}{ Code } \\
\hline & & & 1 & & 2 & 3 \\
\hline & Fixe & d Ratio & 4 & & 5 & 6 \\
\hline & Vari & able Ratio & 7 & & 8 & 9 \\
\hline \multirow[t]{3}{*}{$\mathbf{K} 2$} & \multicolumn{6}{|c|}{ Type of task selected (as coded below): } \\
\hline & Code & Task & & & & \\
\hline & $\begin{array}{l}1 \\
2 \\
3 \\
4\end{array}$ & $\begin{array}{l}\text { Simple addit } \\
\text { Simple subtr } \\
\text { Simple mult } \\
\text { Simple divisi }\end{array}$ & $\begin{array}{l}n \\
\text { tion } \\
\text { ication }\end{array}$ & $\begin{array}{ll}5 & \mathrm{P} \\
6 & \mathrm{P} \\
7 & A \\
8 & \mathrm{C}\end{array}$ & $\begin{array}{l}\text { Position le } \\
\text { Position le } \\
\text { Absolute } n \\
\text { Complex } t\end{array}$ & $\begin{array}{l}\text { g-left } \\
\text { g-right }\end{array}$ \\
\hline
\end{tabular}




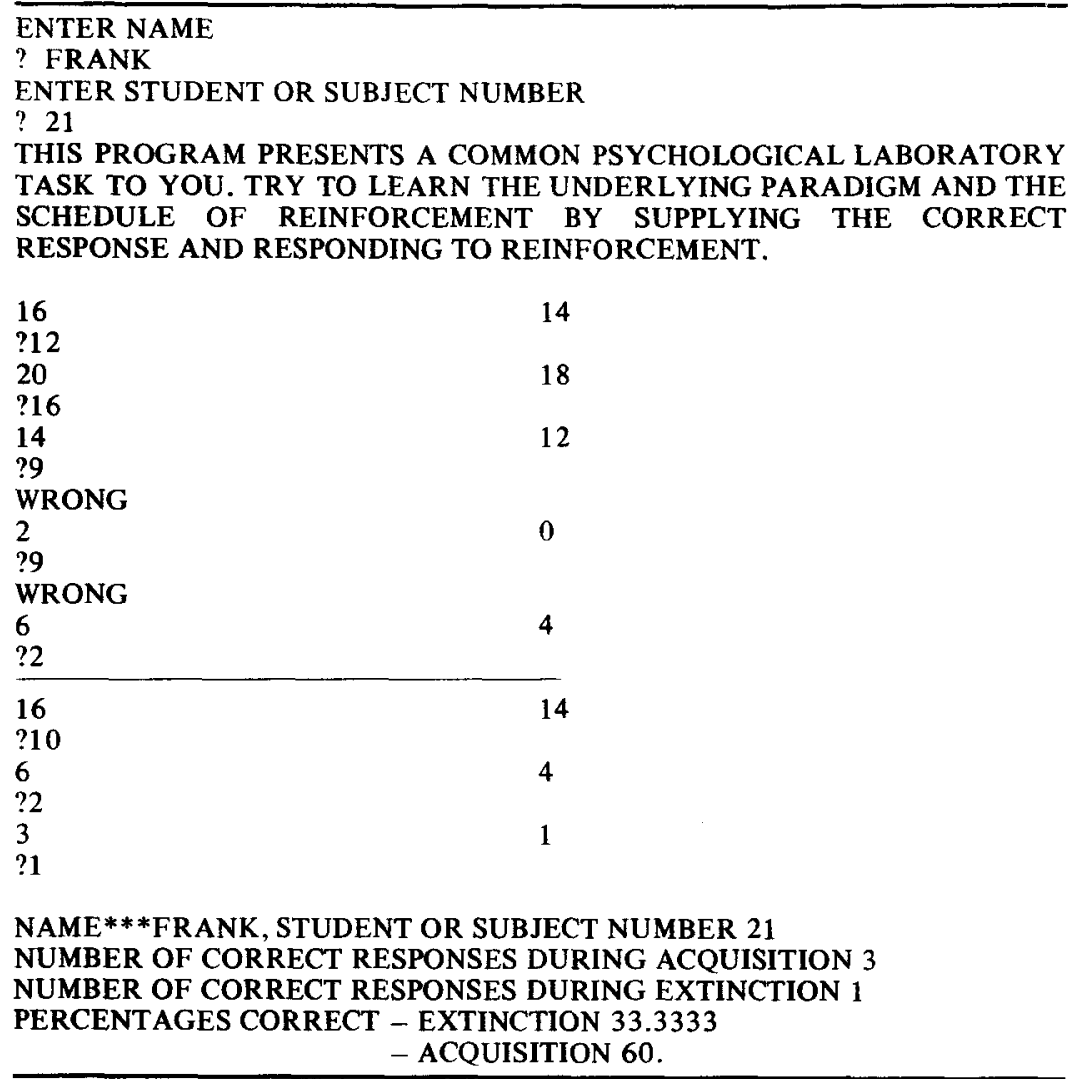

Figure 1. An example of a run of the ALL program for a (fictitious) student who receives continuous negative reinforcement for errors on a simple serial (subtraction) task. The five trials above the line are acquisition trials; those below the line are extinction trials.

prior to running the first research subject. When the research subject enters, the program requests his name and student number. Following this, an introductory message is printed and the task is initiated. The student responds and the appropriate type of reinforcement is meted out according to the selected schedule. The process continues until the student has obtained the required number of acquisition trials. Next the procedure is continued without reinforcement until the specified number of extinction trials is reached. A sample run for a hypothetical student is shown in Figure 1. The run is obviously illustrative. It involves only five acquisition trials and three extinction trials.

After the student has completed the requisite number of trials, the bookkeeping routine prints out the student's name and number and a summary of the number and percentage of correct responses during both acquisition and extinction (see Figure 1). This module also updates the file to be printed for the instructor and spaces the paper to allow the student to remove and carry off his record. Typically, students are run after the segment shown in Figure 1.

When there are no further subjects to be run, the instructor enters "LAST SUBJECT" (unnecessary when a specified number of subjects have been run). This leads to printing of the summary information on all the research subjects employed in the study. Such summary information for a hypothetical group of students is shown in Figure 2.

\section{PROGRAMMING DETAILS AND AVAILABILITY}

The version of ALL described here is written in timesharing BASIC and has been run on an IBM 370/145 computer. Its immediate ancestors have been used on

?LAST SUBJECT

BASIC DESCRIPTIVE STATISTICS

MEANS

STD. DEVIATION

VARIANCE

FRANK

JONES

MARGIE

23

24

ACQUISITION

EXTINCTION

TIME 1 SECS.

Figure 2. An example of the summary output for instructors from the ALL program. 
both IBM 360/50 and CDC 6400 machines. The program is written as a number of modular routines which, despite the absence of a standard for the BASIC language, appear to be reasonably transportable. The program involves about 350 lines and occupies about $16.4 \mathrm{~K}$ bytes of file storage.

A third version of ALL, consisting of the modules employed in the version described here plus several other modules, is also available. It is expected that this version will be more appealing to users with some programming skill and with limitations on storage allocations at their facilities. The modular structure allows for on-site merging of only those routines actually required during a particular computer run. The additional modules consist of a number of other tasks and a statistical routine. The statistical routine provides for the computation of various univariate (e.g., median, skewness index) and bivariate (e.g., correlation between acquisition and extinction performances) statistics. The additional task routines include choosing the largest (or smallest) number from the stimuli presented and a maze learning task. Another modification currently being added to this version will lead to research designs involving a "random effects" independent variable. For example, the researcher will have the option of specifying the levels of fixed-ratio partial reinforcement to be used in his design or of having the computer randomly select these levels.

A variety of other changes, potentially useful in certain settings, have been contemplated. Although the authors may eventually incorporate some of these alterations, they are briefly described here with the hope that they may be of more immediate benefit to interested users.

Clearly, a language more flexible than BASIC would have a number of advantages. First, certain aspects of the coding could be simplified through the use of statements allowing multiple branches. Second, using a language with more output flexibility in terms of computer graphics and a cathode-ray tube (CRT) would allow the inclusion of some discrimination learning tasks. Another possible set of tasks might involve concept learning. Thus, for example, one task might involve the choice of vowels (or consonants) from letter sets. In addition, options for various criteria for acquisition and extinction might be built into any of these versions.

ALL is available on both paper tape and microfiche. The documentation and program can be obtained at a cost of $\$ 5$ from: Sally A. Flanik, Department of Psychology, Howard University, Washington, D.C. 20059.

Please specify whether paper tape, microfiche, or hard copy only is desired and make checks payable to Sally A. Flanik. Unless another version of ALL is specified, the fixed version described here will be sent. That's ALL.

\section{REFERENCE NOTE}

1. Castellan, N. J., Jr., Bailey, D. E., Lehman, R. S., Start, B. J., \& McClelland, G. The current use of computers in instruction in psychology (Report from the Ad Hoc Curriculum Advisory Committee in Psychology). Iowa City, Iowa: CONDUIT, 1976.

\section{REFERENCES}

BEWLEY, W. L. The use of time-shared terminals in a human learning course. Proceedings of the Fourth Conference on Computers in the Undergraduate Curricula. Claremont, Calif: The Claremont Colleges, 1973.

BEwLEY, W. L. Cognitive psychology: A computer-oriented laboratory manual. Hanover, N.H: Project COMPUTe, 1974.

GreEN, B. F., JR. The use of time-shared terminals in psychology. Behavior Research Methods \& Instrumentation, 1972, 4, 51-55.

Lehman, R. S., Stark, B. J., \& Young, K. C. Computer aids in teaching statistics and methodology. Behavior Research Methods \& Instrumentation, 1975, 7, 93-102.

MaIN, D. A computer simulation approach for teaching experimental design. Proceedings of the 79th Annual Convention of the American Psychological Association. Washington, D.C: American Psychological Association, 1971.

Scholz, K. W. Computerized process control in behavioral sciences research. Behavior Research Methods \& Instrumentation, 1972, 4, 203-208. 